\title{
Perfil morfológico e velocidade em atletas de futsal em relação à posição de jogo
}

\section{Artigo Original}

\section{Kelly Samara da Silva \\ Professora de Educação Física \\ ksilvajp@yahoo.com.br}

Francisco Martins da Silva

Professor da Universidade Federal da Paraíba

Laboratório de Estudos e Pesquisas em Treinamento - LEPET

fmsilva@openline.com.br

SILVA, K.S.; SILVA, F.M. Perfil morfológico e velocidade em atletas de futsal em relação à posição de jogo. Fitness \& Performance Journal, v.3, n.4, p.218-224, 2004.

Resumo: Atualmente a maioria dos esportes vem apresentando características morfológicas e neuromotoras específicas às suas necessidades, tornando-se evidente que essas variáveis influenciam no desempenho dos atletas. O presente estudo objetivou identificar o perfil dos jogadores de Futsal das categorias infantil e juvenil do estado da Paraíba através das variáveis antropométricas, composição corporal e velocidade, relacionando-as com as posições de jogo, e observar as diferenças entre as posições de jogo por categoria. Fizeram parte desse estudo as três equipes finalistas do Campeonato Paraibano 2002 nas referidas categorias, onde foram avaliados 68 jogadores, sendo 34 infantis com média de idade de 13,4 anos $\pm 0,7$; e 34 juvenis com idade de 16,3 anos $\pm 1,01$. Os indicadores analisados foram estatura, peso corporal, dobras cutâneas e o teste de 30 metros. Os resultados foram analisados através do teste Turkey HSD e o teste " $\mathrm{t}$ " de Student para variáveis independentes, apontando diferenças significativas $(p<0,05)$ em todas variáreis analisadas; em relação a posição de jogo, apenas os goleiros se diferenciaram dos demais jogadores, exceto na velocidade.

Palavras-chave: Perfil morfológico; Velocidade; Futsal.

Endereço para correspondência:

Rua Jaime Gomes de Barros, 94 - Água Fria - João Pessoa - PB - CEP 58074-080

\begin{tabular}{|l|l|l|}
\hline 3 & 4 & $218-224$ \\
\hline
\end{tabular}




\section{Morphologic profile and speed of sutsal players with regard to play- ing position}

Nowadays the majority of sports are introducing morphologic traits and particular motor each other your own necessities becoming evident that these varieties influence in fulfillment of athletes. Just now was attended a learning about Futsal players childish and youthful categories of Paraíba-Brasil. Through varieties anthropometries, body composition and speed thus relating with positions for categories. Three teams did part of this learning besides they were championship finalist paraibano 2002 that in this categories were evaluated sixty-eight players, been thirty-four childishes average age of 13,4 years old $\pm 0,7$ and thirty-four youthful average age 16,3 years old $\pm 1,01$. The indicators analyzed were stature, corporal weight, fold coetaneous and the test of 30 meters. The results were analyzed through test Turkey HSD and a test " $\mathrm{t}$ " of Student to independent varieties showing differences meanings in all of analyzed varieties and regarding play position only goalkeepers differentiated of other players, bus speed.

Keywords: Morphologic Profile; Speed; Futsal.
Perfil morfológico y velocidad en atletas de futsal con relación a la posición en el juego

Actualmente, la mayoría de los deportes presenta características morfológicas y neuromotoras específicas a sus necesidades, resaltando que esas variables influyen en el rendimiento de los atletas. El presente estudio tuvo el objetivo de identificar el perfil de los jugadores de las categorías infantil y juvenil de futsal del estado de Paraíba (Brasil) a través de las variables antropométricas, composición corporal y velocidad, relacionándolas con las posiciones del juego, de acuerdo con la categoría. Participaron en ese estudio los tres equipos finalistas del campeonato del estado de Paraíba en 2002 en las referidas categorías, en las que se han evaluado 68 jugadores: 34 infantiles con promedio de edad de 13,4 años $\pm 0,7$; y 34 juveniles con edad de 16,3 años $\pm 1,01$. Los indicadores analizados fueron estatura, peso corporal, pliegas cutáneas y la prueba de 30 metros. Se han analizado los resultados a través de la prueba Turkey HSD y la prueba " $t$ " de Student para variables independientes, que identificaron diferencias significativas $(p<0,05)$ en todas las variables analizadas. Con relación a la posición en el juego, sólo los arqueros se han diferenciado de los demás jugadores, con excepción de la velocidad.

Palabras clave: Perfil morfológico; Velocidad; Futsal

\section{INTRODUÇÃO}

interesse em conhecer as quantidades dos diferentes componentes corporais (massa muscular, massa óssea, víceras e gordura corporal) e suas relações com o rendimento esportivo surge da necessidade de obter informações precisas e relevantes acerca dos padrões estruturais e determinantes da performance, enquanto fatores que facilitam e/ou dificultam o desempenho.

Os esportes competitivos assumem características peculiares em relação ao desenvolvimento morfológico e funcional do atleta, a exemplo do excesso de gordura corporal que, além de se constituir numa sobrecarga adicional durante um jogo, segundo WILMORE \& COSTILL (2001), também exerce forte influência sobre o desempenho em velocidade, endurance, equilíbrio, agilidade e potência.

Outro fator complicador seria o baixo peso corporal em função da deficiência de massa isenta de gordura comprometendo também o desempenho atlético, à medida que, a maximização da massa isenta de gordura favorece aos esportes que exigem força, potência e resistência muscular. Logo, tanto o excesso de gordura quanto a deficiência de massa magra associam-se à redução do desempenho atlético em esportes velozes.

Ao caracterizar o Futsal como um esporte de alta exigência de fatores físicos, técnicos e táticos, justifica-se a necessidade de desenvolver condições específicas, capazes de promover atitudes rápidas e precisas na execução de seus gestos motores. A especificidade, somada à atratividade deste esporte, largamente, praticado em clubes, escolas e instituições que apóiam e participam de torneios e campeonatos em diversas categorias, têm contribuído significativamente para à evolução e o crescente reconhecimento do Futsal na sociedade.
De acordo com a Confederação Brasileira de Futsal (CBFS), as competições oficiais só acontecem a partir das categorias infantil e juvenil, fases em que os adolescentes estão entrando no processo de aceleração do desenvolvimento geral, influenciado pela puberdade. Já as categorias inferiores participam de competições regulamentadas por quase todas as federações estaduais (MACHADO \& GOMES, 2001).

Diferentemente do futebol, o futsal é jogado em uma quadra, com medição oficial de 40x20 de largura, com as equipe disponibilizando cinco jogadores titulares distribuídos nas seguintes posições: goleiro - $G$, central - $C$, ala direita e ala esquerda - A e o pivô - $\mathrm{P}$, além de sete a dez reservas conforme o regulamento da competição.

goleiro tem a função de defender e também de atacar, de acordo com as regras atuais. $O$ central é conhecido como o armador e principal marcador da equipe, os alas são responsáveis pelo padrão de movimentação do jogo e pela finalização e o pivô é aquele jogador habilidoso e rápido na função de prender, distribuir e finalizar.

Essas características, certamente, exigem capacidades físicas e padrões motores específicos. Estudos desenvolvidos por ARAÚJO et al. (1996) determinam que o Futsal é uma modalidade de caráter intermitente, sendo caracterizado pelo predomínio de esforços intensos e curtos com atuação metabólica dos três sistemas energéticos (anaeróbio alático, lático e aeróbio), com níveis de predominâncias diferenciados.

Segundo MACHADO \& GOMES (2001), a resistência aeróbia representa a essência de uma partida de Futsal, sendo a 
resistência anaeróbia lática notada nas alternâncias entre as ações ofensivas e defensivas, e a resistência anaeróbia alática evidenciada, principalmente, durante as ações técnicas. Neste sentido, a velocidade é de fundamental importância para atletas que competem em esportes com ações rápidas, como as que se apresentam no Futsal, como os deslocamentos, os dribles, as fintas e os chutes.

Sabe-se também que as características antropométricas e a composição corporal influenciam no desempenho de um jogador de Futsal. Dessa forma, torna-se necessário não apenas estudar o perfil morfológico desses atletas, mas também relacioná-lo com as posições de jogo, em razão do tipo de exigência a que o atleta é submetido.

Outra preocupação atual se volta para o estudo do perfil do atleta considerando a sua posição de jogo. É importante analisar o atleta dentro de suas condições reais "específicas", pois a estrutura morfológica e as capacidades de desempenho físico exercem papéis diferenciados e importantes no desempenho.

Considerando o exposto acima somado à carência de estudos específicos na área, definiu-se como objetivo desse estudo:

- Traçar os perfis antropométricos e de velocidade dos jogadores de Futsal das categorias infantil e juvenil, relacionando-os com as funções desempenhadas em quadra;

\section{REFERENCIAL TEÓRICO}

A composição corporal de atletas tem sido investigada por vários estudiosos envolvidos com o rendimento esportivo. À medida que as exigências competitivas aumentam, também aumentam a preocupação em atingir valores desejáveis de gordura corporal e massa muscular ideais à necessidade do esporte.

Atualmente, a técnica antropométrica é um dos procedimentos de maior aplicabilidade para estudos de medidas externas das dimensões corporais (GUEDES \& GUEDES, 1998). Este método é um dos mais difundidos e utilizados no Brasil, uma vez que utiliza equipamentos de baixo custo e de simples execução (LOPES \& PIRES NETO, 1996).

A antropometria permite medir o crescimento através da avaliação da estatura e do peso corporal, bem como a quantidade e o pa- drão de distribuição da gordura corporal através da espessura das dobras cutâneas e somatória das diferentes regiões anatômicas (GUEDES \& GUEDES, 1998; COSTA, 2001).

Em relação ao cálculo do percentual de gordura, são poucas as equações propostas para crianças e jovens, dentre as existentes destacam-se as equações que receberam maior aceitação, como BOILEAU (1985); SLAUGHTER et al. (1988); GUEDES \& GUEDES (1998); HEYWARD \& STOLARCZIK (2000). A somatória de dobras cutâneas pode acontecer em situações distintas, com a somatória de dobras dos membros e do tronco, separadamente, além de outras combinações, como por exemplo, a somatória de oito ou nove dobras (COSTA, 2001).

Nos últimos tempos, a massa isenta de gordura (MIG) tornouse, também, um forte aliado na busca pelo desempenho atlético e controle da performance. Estudos desenvolvidos por FILIN \& VOLKOV (1998) constataram que, durante os primeiros 15 anos de idade, o peso dos músculos aumenta em aproximadamente $9 \%$, e nos 2 a 3 anos seguintes (15-17/18anos) aumentam em $12 \%$.

Em relação a velocidade, entendida como a capacidade de realizar ações motoras com máxima intensidade num curto espaço de tempo, é bastante influenciada pela quantidade de MIG (HAHN, 1988; MANSO et al., 1996; ADELINO et al, 1999) e, também, pela idade, sexo, antropometria, qualidade da técnica esportiva, talento, dentre outros (GROSSER, 1996). O seu desenvolvimento se dá em função da idade biológica e do desenvolvimento da criança, portanto crianças e jovens precoces desenvolvem a sua força mais cedo e estão aptos a atingir maiores velocidades em relação àquelas de desenvolvimento retardado (MANSO et al., 1996; WEINECK, 1999).

Para MANSO et al. (op cit), o tempo de reação e a velocidade cíclica alcançam os mesmos valores em crianças em idades prepuberal e puberal, quando comparadas a adultos, em função da enorme plasticidade que tem o sistema nervoso central (SNC) nesta fase. Para MOSKATOVA (1998), a idade de desenvolvimento máximo da explosão e da velocidade nos 30 metros para os jovens futebolistas está entre 11-13 anos.

WILMORE \& COSTILL (2001), em estudos com crianças e jovens, verificaram que os meninos apresentaram um desenvolvimento gradativo no tempo (s) do teste de velocidade à medida que a idade evoluía, de forma que entre os 13 e 14 anos o $T=7,0-7,5$ segundos e entre os 15 a 17 anos o $T=6,5-6,8$ segundos. Em

Quadro 1 - Resultados médios das variáveis estudadas por categoria

\begin{tabular}{|c|c|c|c|c|c|c|c|c|}
\hline$C$ & PC & $\mathrm{E}$ & $\% G$ & MIG & $\Sigma T$ & $\Sigma M$ & $\Sigma 8$ & V \\
\hline 1 & $53,0 \pm 11,8$ & $163,7 \pm 8,9$ & $16,7 \pm 5,9$ & $43,7 \pm 7,5$ & $41,5 \pm 24,4$ & $42,6 \pm 15,1$ & $84,1 \pm 37,8$ & $6,8 \pm 0,4$ \\
\hline $\mathrm{J}$ & $62,1 \pm 8,3$ & $172,7 \pm 6,3$ & $15,5 \pm 3,4$ & $52,4 \pm 5,9$ & $40,3 \pm 13,6$ & $33,4 \pm 7,0$ & $73,6 \pm 19,2$ & $7,3 \pm 0,3$ \\
\hline DifX & $-9,1$ & $-9,1$ & 1,2 & $-8,6$ & 9,3 & $-9,9$ & 1,2 & $-0,6$ \\
\hline P* & ,001 &, 000 & ,330 &, 000 & ,003 &, 000 & ,808 & ,000 \\
\hline
\end{tabular}

Categorias (C); Diferença de Média (DifX) e $p<0,05\left(P^{*}\right)$. 
relação à MIG, apresentaram maiores proporções entre os 14 e 15 anos, com aumento gradativo até os 20 anos; para a MG, os resultados foram próximos até 15 anos.

SILVA (1999) realizou um estudo em Pelotas/RGS com quatro equipes de Futsal juvenil com média de idade de 16 anos, os quais foram avaliados em relação ao peso corporal, à estatura, ao percentual de gordura e à massa magra. Os valores não apresentaram significância em relação à posição de jogo. No entanto, quando comparados por equipes, apenas a adiposidade se diferenciou em um clube em relação aos demais.

KISS et al. (1999) apresentaram resultados antropométricos (peso corporal e estatura) de uma equipe de Futsal com 16 indivíduos. Os resultados apontaram uma estatura de $177,6 \pm 3,3 \mathrm{~cm}$ e peso corporal de 76,4 $\pm 6,2 \mathrm{~kg}$. PEREIRA \& SILVA (2000) estudaram o perfil dos 12 atletas jogadores de Futsal da seleção brasileira universitária, com idade entre 22 a 27 anos, encontrando os seguintes resultados: massa magra $61,55 \mathrm{~kg}$, massa gorda $9,3 \mathrm{~kg}$ e velocidade nos 30 metros de 4,18 segundos.

SOUZA (1999) desenvolveu um estudo com jogadores de futebol de várias categorias relacionando a posição de jogo. Os resultados apontaram o aumento do peso corporal e da estatura e a redução do percentual de gordura, com a mudança de categoria.

SILVA et al. (1997) e LEY et al. (2002) estudaram as diferenças antropométricas e metabólicas entre jogadores de futebol das categorias juvenil, juniores e profissional. Ambos estudos constataram diferenças significativas nas variáveis antropométricas no grupo juvenil, quando comparado aos demais grupos.

\section{MATERIAL E MÉTODOS}

Segundo THOMAS \& NELSON (2002), a pesquisa descritiva é um tipo de estudo de status utilizado para caracterizar resoluções de problemas por meio de observação, análise e descrições objetivas e completas. A pesquisa descritiva de cunho desenvolvimental, através de corte transversal, possibilita a investigação do crescimento e a composição corporal dos sujeitos em diferentes grupos etários.

\section{Amostra}

A população desse estudo foi constituída pelas três equipes finalistas $\left(1^{\circ}, 2^{\circ}\right.$ e $3^{\circ}$ lugares) do Campeonato Paraibano 2002, nas categorias Infantil (I) e Juvenil (J). No total, foram avaliados 68 jogadores na faixa etária dos 13 aos 18 anos, sendo 34 da categoria infantil, com média de idade de 13,4 anos \pm 0,7meses, e 34 da categoria juvenil, com idade de 16,3 anos $\pm 1,01$ anos.

Considerando a posição de jogo, a amostra apresenta a seguinte distribuição: 8 goleiros (4 infantis e 4 juvenis), 20 centrais, 20 alas e 20 pivôs, distribuídos 10 para cada categoria.

\section{Instrumentos e Procedimentos}

Os atletas foram avaliados através de medidas do peso corporal, estatura, dobras cutâneas e um teste de 30 metros. Para facilitar o processo de coleta dos dados, montou-se um grupo de quatro pessoas com experiência na área de avaliação e participação em projetos desenvolvidos pelo Laboratório de Estudos e Pesquisas em Treinamento (LEPET/UFPB), de forma que uma pessoa fez a aferição do peso corporal e da estatura; duas pessoas foram responsáveis pelas dobras cutâneas e outra pela execução do teste de velocidade.

Peso corporal foi medido em uma balança Filizola, com precisão de 100g; e a estatura foi determinada através de uma fita métrica com a precisão de $0,1 \mathrm{~cm}$ fixada perpendicularmente a uma parede lisa (sem rodapé) no momento de inspiração máxima executada pelo avaliado (GORDON et al.,1988).

Quadro 2 - Variáveis estudadas por categoria e posição de jogo

\begin{tabular}{|c|c|c|c|c|c|c|c|c|c|}
\hline PJ & $\mathrm{C}$ & PC & $E$ & $\% G$ & MIG & $\Sigma \mathrm{ET}$ & $\Sigma D M$ & $\Sigma 8 D$ & V \\
\hline \multirow{2}{*}{ G } & I & $71,7 \pm 15,4$ & $170,5 \pm 4,7$ & $29,0 \pm 6,7$ & $50,1 \pm 7,4$ & $96,8 \pm 35,2$ & $71,7 \pm 21,2$ & $168,5 \pm 52,0$ & $6,4 \pm 0,3$ \\
\hline & $\mathrm{J}$ & $77,1 \pm 5,0$ & $184,0 \pm 1,8^{*}$ & $17,4 \pm 2,4^{*}$ & $63,6 \pm 2,4^{*}$ & $47,8 \pm 15,2 *$ & $39,6 \pm 7,7^{*}$ & $87,4 \pm 21,6^{*}$ & $7,4 \pm 0,2 *$ \\
\hline \multirow{2}{*}{ C } & I & $48,6 \pm 10,1$ & $160,2 \pm 9,9$ & $14,6 \pm 3,3$ & $41,4 \pm 7,7$ & $34,9 \pm 6,1$ & $38,4 \pm 3,8$ & $73,3 \pm 7,4$ & $6,7 \pm 0,4$ \\
\hline & $\mathrm{J}$ & $63,3 \pm 5,6^{*}$ & $172,0 \pm 4,4^{*}$ & $16,9 \pm 4,2$ & $52,4 \pm 3,2^{*}$ & $42,8 \pm 14,8$ & $36,0 \pm 7,1$ & $78,9 \pm 20,1$ & $7,2 \pm 0,3^{*}$ \\
\hline \multirow{2}{*}{ A } & I & $51,5 \pm 8,7$ & $164,0 \pm 9,3$ & $15,6 \pm 2,9$ & $43,5 \pm 7,7$ & $32,4 \pm 6,9$ & $38,6 \pm 8,8$ & $71,0 \pm 11,5$ & $6,8 \pm 0,3$ \\
\hline & $\mathrm{J}$ & $57,3 \pm 6,1$ & $171,0 \pm 5,8$ & $14,0 \pm 3,2$ & $49,2 \pm 4,8$ & $36,4 \pm 13,7$ & $30,4 \pm 7,0 *$ & $66,8 \pm 19,9$ & $7,3 \pm 0,3^{*}$ \\
\hline \multirow{2}{*}{$P$} & I & $51,6 \pm 8,5$ & $164,0 \pm 8,1$ & $14,8 \pm 3,8$ & $43,8 \pm 6,7$ & $35,0 \pm 11,7$ & $39,4 \pm 12,9$ & $74,4 \pm 23,9$ & $6,9 \pm 0,4$ \\
\hline & $\mathrm{J}$ & $59,3 \pm 5,7^{*}$ & $170,1 \pm 4,5$ & $14,7 \pm 2,4$ & $50,5 \pm 4,3^{*}$ & $38,2 \pm 11,9$ & $30,9 \pm 4,6$ & $69,1 \pm 14,4$ & $7,4 \pm 0,2^{*}$ \\
\hline \multicolumn{10}{|c|}{ Significância dos resultados por posição de jogo } \\
\hline \multirow{3}{*}{ G } & $\mathrm{C}$ & * & $*$ & * & * & * & * & * & - \\
\hline & A & * & * & * & * & $*$ & $*$ & $*$ & - \\
\hline & $P$ & * & * & * & * & * & * & * & - \\
\hline \multirow{2}{*}{$\mathrm{C}$} & A & - & - & - & - & - & - & - & - \\
\hline & $\mathbf{P}$ & - & - & - & - & - & - & - & - \\
\hline
\end{tabular}

PJ- posição de jogo(G-goleiro; C-central; A- alas; P- pivô) ; C- categorias (I- infantil; J- juvenil) ; p* (<0.05); p > 0.05(-). 


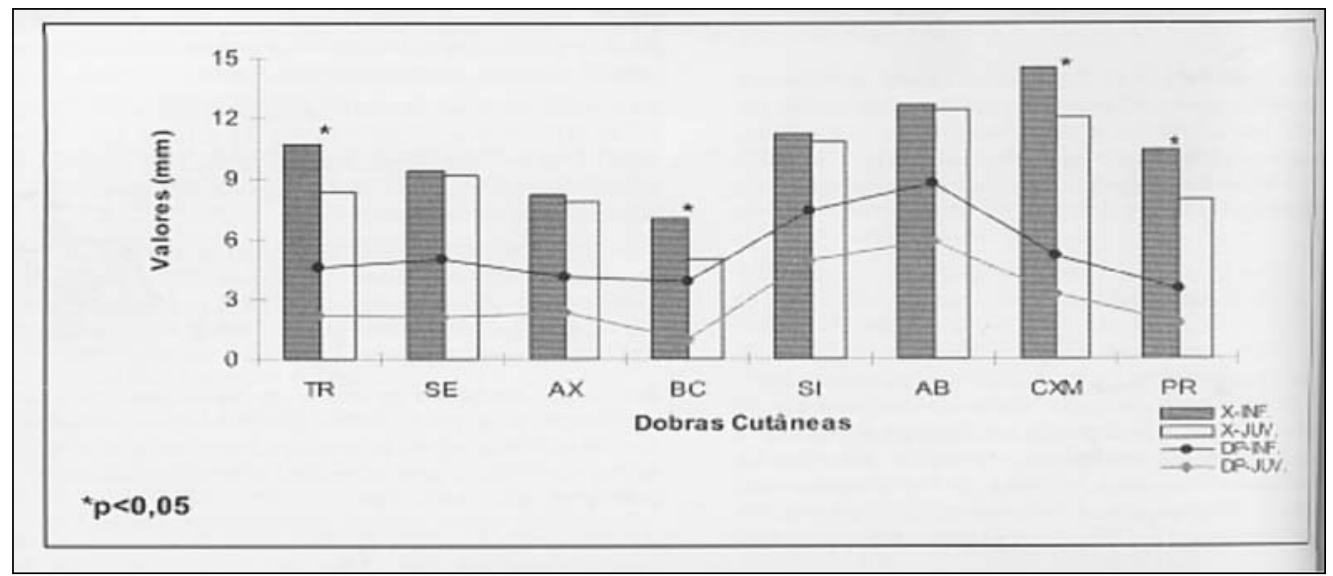

Figura 1 - Comportamento das dobras cutâneas por categoria

${ }^{*} p<0,05$
A composição corporal foi avaliada através da mensuração de 08 dobras cutâneas (triciptal, subscapular, suprailíaca, biciptal, axilar média, abdominal, coxa medial e panturrilha medial), utilizando um plicômetro modelo Harpenden pela pressão constante de $10 \mathrm{~g} / \mathrm{mm}^{2}$ e fácil manipulação. As medidas foram aferidas no hemisfério direito do corpo do avaliado, através do pinçamento com os dedos polegar e indicador, num total de 3 medições realizadas consecutivamente em espaço de 4 segundos, sendo registrado o valor médio.

Para avaliação do percentual de gordura foi utilizada a equação de BOILEAU et al. (1985): \%G=1,35 (STR + SE) - 0,012 (STR $+\mathrm{SE})^{2}-4,4$, desenvolvida através de medidas de referências de modelos multicomponentes, sendo o mais utilizado para esta população.

A massa isenta de gordura foi obtida através da equação proposta por RIBEIRO (1998), determinando a massa corporal gorda (MG) = massa corporal $(\mathrm{kg}) \times$ gordura corporal (\%)/ 100 e a massa corporal isenta de gordura (MIG) = massa corporal $(\mathrm{kg})$ - massa corporal gorda $(\mathrm{kg})$.

Realizou-se também a somatória de dobras para observar a distribuição da gordura corporal geral e em diferentes regiões do corpo. Para a região do tronco foram utilizadas as dobras $S E, S I, A X$ e $A B$, e para os membros foram consideradas as dobras TR, BC, CXM e PR.

A velocidade foi avaliada por uma única pessoa com experiência de três anos no manuseio de cronômetro, através do teste dos 30 metros, com saída parada e corrida no sentido retilíneo, realizado em quadra de jogo com medidas variando entre 36 a 40 metros de comprimento, com uma zona de desaceleração de 10 a 12 metros. Foram realizadas duas tentativas consecutivas com um intervalo de 3 minutos, sendo considerada a melhor. $\bigcirc$ resultado foi exposto em metros por segundos $(\mathrm{m} / \mathrm{s})$.

Todos os testes foram realizados no período noturno no ginásio de treinamento das equipes com datas e horários estabelecidos pelos treinadores. A duração total da coleta de dados foi de aproximadamente duas semanas e aconteceu em paralelo ao segundo turno do Campeonato Paraibano.

\section{Tratamento Estatístico}

Para análise dos resultados utilizou-se a estatística descritiva (média, desvio padrão); o teste Tukey HSD para comparações múltiplas entre as posições de jogo por categoria; e o teste † para variáveis independentes, visando observar as diferenças por posição de jogo.

\section{RESULtAdOS E DISCUSSÕES}

A figura abaixo ilustra o comportamento das dobras cutâneas por categorias, onde observa-se um declínio da proporção das dobras à medida que a categoria avança, com reduções significativas

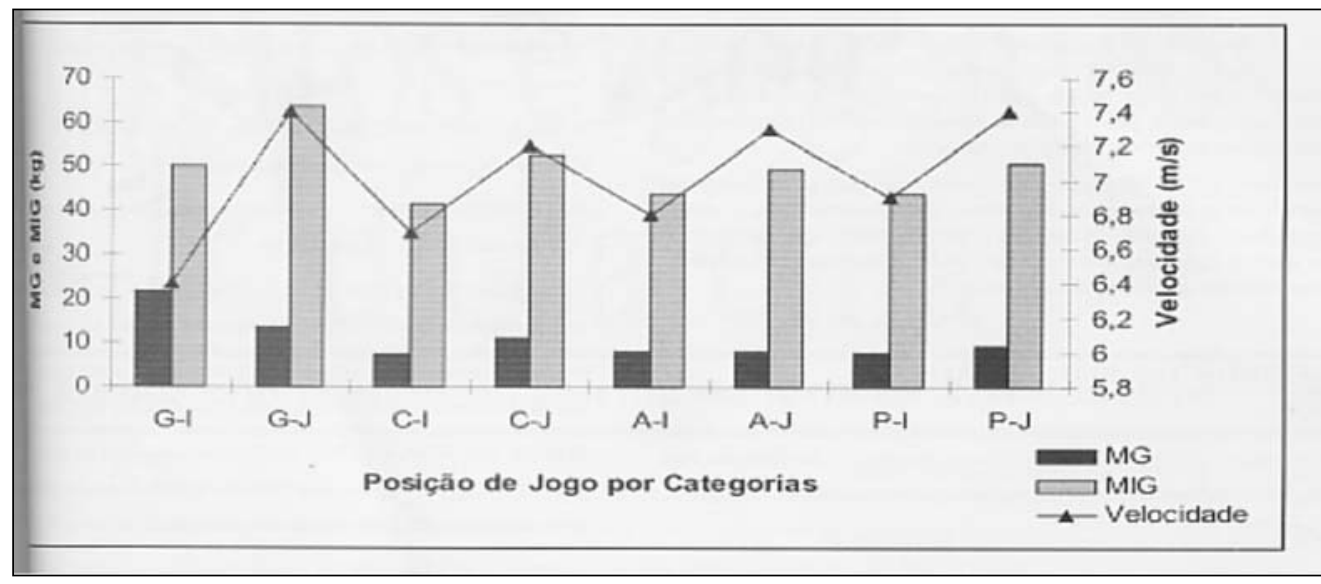

Figura 2 - Composição corporal e a velocidade por posição de jogo 
nas dobras cutâneas localizadas na região dos membros, quando comparamos os jogadores juvenis aos infantis.

Em relação a somatória das dobras cutâneas, observou-se que o grupo infantil apresentou resultados superiores e significativos tanto para a åT quanto para åM, quando comparado ao juvenil. Este dado constata o decline da gordura corporal com a evolução da idade, outra constatação foi o aumento da MIG, com diferença de média de $8,6 \mathrm{~kg}$ em relação ao infantil, conforme o quadro abaixo.

Um outro fator positivo no juvenil foi o desenvolvimento da velocidade média com diferença de 0,6 centésimos em relação ao infantil, o que viabiliza a influência entre o aumento da MIG e a melhora na explosão (GROSSER, 1996), além de outros fatores como a idade e a treinabilidade (WEINECK, 1999).

A Figura 2 ilustra a relação da composição corporal com a velocidade, considerando a posição de jogo entre as categorias. Em relação a composição corporal observou-se tanto no juvenil quanto no infantil que aqueles que apresentaram menor quantidade de massa gorda foram os que obtiveram uma maior velocidade.

Essas alterações são justificadas pelo processo de crescimento e maturação presentes nesta faixa de idade com fases susceptíveis ao aumento da testosterona e desenvolvimento da estrutura corporal (WILMORE \& COSTILL, 2001 ; FILIN \& VOLKOV, 1998).

O Quadro 2 descreve as diferenças consideráveis nos resultados apresentados, considerando as posições de jogo e comparandoas entre categorias. Dessa forma observa-se que os goleiros juvenis apresentaram resultados significativos em todas as variáveis, exceto no peso corporal, quando comparado aos infantis.

Já os centrais e os pivôs juvenis apresentaram valores superiores e significativos para o peso corporal, MIG e estatura em relação aos infantis, enquanto que os alas juvenis apresentaram reduções significativas na somatória de dobras dos membros. Em relação a velocidade, a categoria juvenil apresentou diferenças significativas em todas as posições quando comparadas à infantil.

Analisando a velocidade, verifica-se que os jogadores de linha infantis e juvenis apresentaram um comportamento linear de desenvolvimento, de forma que os centrais são os jogadores menos velozes, os alas são os jogadores de velocidade intermediária e os pivôs, os mais velozes.

Outra preocupação desse estudo foi o de verificar, separadamente, por categoria se os resultados caracterizados por posição de jogo eram significativos em relação às outras posições. De acordo com os resultados verificou-se que apenas os goleiros apresentaram resultados significativos nas variáveis estudadas, exceto na velocidade, quando comparado as demais posições.
Com isso, é possível afirmar que os jogadores de linha de futsal não apresentaram características específicas nas variáveis estudadas em relação a posição de jogo confirmando o estudo do SILVA et al. (1999).

\section{CONCLUSÃO}

De acordo com os resultados obtidos, conclui-se que o avanço da categoria influencia diretamente no desenvolvimento morfológico e na velocidade em função das alterações morfofuncionais procedidas pelo processo de crescimento, maturação e, também, do treinamento.

Em relação às posições de jogo, os dados apontaram significância apenas para os goleiros, principalmente os juvenis, quando comparadas às demais posições. Com isso, constatou-se que os jogadores de linha de Futsal apresentaram um perfil morfológico e velocidades similares.

\section{REFERÊNCIAS BIBLIOGRÁFICAS}

ADELINO, J.; VIEIRA J.; COELHO, O.: Treino de Jovens: O Que Todos Precisam Saber! Lisboa: Centro de Formação Desportiva, Secretaria do Estado do desporto: 1999.

ARAÚJO, T.L. et al. Demanda fisiológica durante o jogo de futebol de salão, através da distância percorrida. Revista da Associação dos Professores de Educação Física de Londrina, 11(19): 12-20, 1996.

BOILEAU, R.A.; LOHMAN, T.G. \& SLAUGHTER, M.H.: Exercise body composition in children and youth. Scandinavian Journal of Sport Sciences, 7:17-27, 1985.

COSTA, R.F.: Composição corporal: Teoria e prática da avaliação. $1^{a}$ ed. Barueri/SP: Manole, 2001.

FILIN, V.P.; VOLKOV, V.M.; Seleção de Talentos nos Desportos. Londrina: Midiograf, 1998.;

GORDON, C.C., CHUMLEA, W.C.; ROCHE, A.F; Stature, recumbert length and weight. In TG Lohman et al., Anthropometric Stardardization Reference Manual. Champaign: Human Kinetics, 3-8, 1988.

GROSSER, M. Entrenamiento de la Velocidad: Fundamentos, Métodos Y Programas. Barcelona: Martínez Roca, 1992.

GUEDES, D.P.; GUEDES, J.E.R.P. Controle do Peso Corporal: Composição Corporal, Atividade Física e Nutrição. Londrina: Midiograf, 1998.

HAHN, E. Entrenamiento Com Niños: Teoria, Práctica, Problemas Específicos. Barcelona: Martínez Roca, 1988.

HEYWARD, V.H. \& STOLARCZYK, L.M. Avaliação da Composição Corporal. São Paulo: Manole, 2000.

KISS, M.A.; BOHME, M.T.S. \& REGAZZINI, M. Cineantropometria, In: Barros, T. \& Ghorayeb, N. O exercício: preparação fisiológica, avaliação médica, aspectos especiais e preventivos. São Paulo: Atheneu, 117-130, 1999.

LEY, R.O.; GOMES, A.C.; MEIRA, A.L.J.; ERICHSEN, A.O.; SILVA, S.G. Estudo comparativo dos aspectos funcionais e de composição corporal entre atletas do futebol de diferentes categorias. Revista Brasileira de Fisiologia do Exerćício, Rio de Janeiro: Sprint, 1(1): 75-87, 2002.

LOPES, A.S.; PIRES, NETO. Composição Corporal e Equações Preditivas da Gordura em Crianças e Jovens. Revista Brasileira de Atividade Física e Saúde, 4: 38-52, 1996.

MACHADO, J.A.; GOMES, A.C. Futsal Metodologia e Planejamento na Infância e Adolescência. Londrina, Midiograf, 2001.

MANSO, J.H.G.; VALDIVIELSO, M.N. E CABELLERO, J.A.R. Bases Teóricas Del Entrenamiento Deportivo: Princípios Y Aplicaciones. Madrid: Ed. Gymnos, 1996.

MOSKATOVA, A.K. Aspectos Genéticos e Fisiológicos no Esporte: Seleção de Talentos na Infância e Adolescência. Rio de Janeiro: Grupo Palestra Sport, 1998.

PEREIRA, P.A.; SILVA, K.S. Perfil dos jogadores de futsal da seleção brasileira universitária, campeã do mundial 2000. In Anais do Simpósio Internacional em Treinamento Desportivo, João Pessoa: ed. Universitária, 2000.

RIBEIRO, J.C.R.D. Composição Corporal, Obesidade e Aptidão Cardiorespiratória: Estudo Descritivo em Crianças de Ambos os Sexos, com Idades Compreendidas entre os 08 e os 16 anos, da Área do Grande Porto. Dissertação. FCDEF. Porto: 1998. 
SILVA, M.F. Estrutura da performance desportiva: um estudo referenciado ao Futsal na categoria juvenil. In I prêmio Indesp de literatura desportiva, Brasília, 1:175-247, 1999.

SILVA, S.G.; PEREIRA, J.L.; KAISS, L.; KULAITIS, A.; SILVA, M. Diferenças Antropométricas e Metabólicas entre Jogadores de futebol das Categorias Profissional, Júnior e Juvenil. Revista Treinamento Desportivo, V II (3): 35-39, 1997.

SLAUGHTER, M.H.; LOHMAN, T.G.; BOILEAU, R.A.; HORSWILL, C.A.; STILMAN, R.J.; VAN LOAN, M.D. \& BEMBEN, D.A. Skinfold equations for estimation of body fatness in children and youth. Human Biology, 60: 709-723, 1988
SOUZA, J. Variáveis Antropométricas, Metabólicas e Neuromotoras de Jogadores de Futebol das Categorias Mirim, Infantil, Juvenil e Júnior e em relação a Posição de Jogo: Um Estudo Comparativo. Revista Treinamento Desportivo, IV (3): 43-48, 1999.

THOMAS \& NELSON. Métodos de pesquisa em atividade física. 3166 ed., Porto Alegre: Artmed, 2002.

WEINECK, J. Treinamento ideal: instruções técnicas sobre o desempenho fisiológico, incluindo considerações específicas de treinamento infantil e juvenil. São Paulo: Manole, 1999.

WILMORE, J.H. \& COSTILL, D.L. Fisiologia do esporte e do exercício. São Paulo: Manole, 2001. 